\title{
Cognitive modeling analysis of decision-making processes in cocaine abusers
}

\author{
JULIE C. STOUT, JEROME R. BUSEMEYER, and ANLI LIN \\ Indiana University, Bloomington, Indiana \\ and \\ STEVEN J. GRANT and KATHERINE R. BONSON \\ National Institute on Drug Abuse, Bethesda, Maryland
}

\begin{abstract}
This article examines the theoretical basis of decision-making deficits exhibited by cocaine abusers in a laboratory decision-making task first described by Bechara, Damasio, Damasio, and Anderson (1994). A total of 12 male cocaine abusers and 14 comparison subjects performed the task, and the cocaine group performed significantly worse than the comparison group. A cognitive modeling analysis (Busemeyer \& Stout, 2002) was used to estimate three parameters that measure importance of the cognitive, motivational, and response processes for determining the observed performance deficit. The results of this analysis indicated, for the first time, that motivational and choice consistency factors, but not learning/memory were mainly responsible for the decision-making deficit of the cocaine abusers in this task.
\end{abstract}

Drug abusers choose the immediate rewards of using drugs despite negative consequences over the longer term. Laboratory-based decision tasks, such as betting and delay discounting tasks, serve as scientific tools to uncover how cognition and motivation interact with contingencies in the environment to create and sustain drug abuse in some individuals (Kirby, Petry, \& Bickel, 1999; Petry, Bickel, \& Arnett, 1998; Rogers et al., 1999). More recently, a simulated gambling task developed by Bechara, Damasio, Damasio, and Anderson (1994, hereafter referred to as the Iowa gambling task) has become very influential for studying decision-making deficits in drug abusers. In this task, participants select cards from four decks that have unknown contingencies. The participants are told to try to maximize their winnings, but because the contingencies of the decks are not explicit, they must sample from the decks to learn the associations between particular decks and the win/loss outcomes. Several studies have now confirmed that drug abusers perform worse on this gambling task than comparison subjects, choosing relatively more from disadvantageous and less from advantageous decks (Bechara \& Damasio, 2002; Bechara, Dolan, \& Hindes, 2002; Grant, Contoreggi, \& London, 2000). Moreover, these findings with drug abusers on the Iowa gambling task are similar to those found with orbitofrontal cortex damaged patients, suggesting that al-

S.J.G. is now with the NIDA Program Office; K.R.B. is now at the Controlled Substances Program of the U.S. Food and Drug Administration. This research was supported by NIDA Grant DA-R01 014119 and by the Intramural Research Program of the National Institute on Drug Abuse. Correspondence concerning this article should be addressed to J. C. Stout, Department of Psychology, Indiana University, 1101 East 10th St., Bloomington IN 47405-7007 (e-mail: jcstout@indiana.edu). terations in the function of the orbitofrontal cortex and its associated circuitry may be important in drug abuse (Bechara \& Damasio, 2002; Grant et al., 2000; London, Ernst, Grant, Bonson, \& Weinstein, 2000).

Decision tasks are complex and have many unobservable components. Thus, failure to perform well on these tasks can be due to any of a number of causes. For example, poor performance on the gambling task may be due to poor learning of the contingencies, to differences in the motivational significance of wins or losses, or even to an impulsive or erratic response style, which can reduce the consistency between one's expectations about the contingencies and the choices one makes. To uncover the sources of performance variations across decision tasks and across individuals, researchers have used variations in the task design - for example, by reversing the patterns of wins and losses (Bechara et al., 2002) to isolate possible explanations of choice behavior. Another approach is to use additional measures, such as indices of personality or cognitive ability, to identify individual differences associated with decision task performance (Bechara \& Damasio, 2002; Bechara et al., 2002; Maddox \& Filoteo, 2001; Mazas, Finn, \& Steinmetz, 2000; Petry et al., 1998). Such approaches have been informative, but their results remain open to alternative interpretations because these measures rely on face validity rather than deductive theoretical evidence.

Mathematical psychologists are developing new theoretical tools to provide rigorous evidence for identifying the psychological processes underlying complex behaviors in cognitive tasks (Busemeyer \& Stout, 2002; Ratcliff, Thapar, \& McKoon, 2001; Riefer, Knapp, Batchelder, Bamber, \& Manifold, 2002; Zaki \& Nosofsky, 2001). For this purpose, simple mathematical models of a target task 
are developed and tested, and the parameters estimated from these cognitive models are used to describe the relative influences of task features on an individual's performance. Thus, individual difference measures of the relevant cognitive processes are extracted from the same performance data that one desires to explain.

Recently, we developed a cognitive model of decisionmaking that reveals the underlying psychological processes influencing performance on the Iowa gambling task (Busemeyer \& Stout, 2002). This initial application was limited to data collected from patients with Huntington's disease, which is a progressive dementing illness. Our cognitive modeling analyses indicated that learning and memory processes, rather than motivational processes, were responsible for the decision-making deficit exhibited by this population. The crucial question addressed in the present article is whether the decision-making deficit exhibited by drug abusers on the Iowa gambling task is caused by the same source or a different source. Surprisingly, we found that a completely different source, based on motivational processes, is responsible for the decisionmaking deficit in drug abusers.

\section{METHOD}

\section{Participants}

As part of a study in the National Institute on Drug Abuse (NIDA) Intramural Program, 14 comparison, or nonabusing (age, $30.0 \pm$ 6.1 years; $100 \%$ male; estimated IQ, $105.0 \pm 7.62$ ), and 12 cocaineabusing (age, $36.9 \pm 10.3 ; 79 \%$ male; estimated IQ, $93.7 \pm 10.3$ ) individuals participated. The cocaine group was older $[t(24)=$ $-3.04, p<.01]$ and had lower IQs $[t(24)=3.22, p<.01]$ than did the comparison group. Exclusion criteria were history of significant medical illness or head trauma with loss of consciousness, $\mathrm{Di}$ agnostic and Statistical Manual (DSM) Axis I diagnosis other than Substance Abuse and Axis II diagnosis other than Borderline or Antisocial Personality Disorder. The cocaine group reported current regular cocaine abuse, and all had past experience with other drugs of abuse (stimulants, depressants, marijuana, opiates). One had a history of alcohol abuse. Their IQs were estimated using the Shipley IQ Scale (Zachary, 1986). These participants were also in a PET study of the gambling task, although the PET results are not included in this report. All participants gave written informed consent.

\section{Procedure}

The participants were tested while seated in a comfortable chair in front of a computer monitor. Four card decks, labeled A, B, C, or $\mathrm{D}$, were displayed on the monitor, and the participants were told to accumulate as much (play) money as possible by picking cards from the decks using the keyboard. The participants also viewed levels of their winnings, shown on the monitor as stacks of coins. ${ }^{1}$

Decks differed with respect to the payoff for each card selection and the frequency and severity of penalties. Each selection from Decks A and B yielded $\$ 100$, and each selection from Decks C and $\mathrm{D}$ yielded $\$ 50$. These winnings were paired with losses on many cards, so that Decks A and B were disadvantageous overall, leading to a net loss of $\$ 250$ per 10 cards, whereas Decks $C$ and $D$ were advantageous overall, leading to a net gain of $\$ 250$ per 10 cards. Decks A and C yielded lower frequency, larger magnitude losses, whereas Decks B and D yielded smaller, more frequent losses (for additional details, see Grant et al., 2000).

\section{Cognitive Modeling of Gambling Task}

Next we will present the expectancy valence model, which was designed to represent the motivational, learning/memory, and choice consistency components of the decision process for the Iowa gambling task. We will also compare this model with two baseline models, which are described below.

The weight given to losses relative to gains, $w$. According to the expectancy valence model, the decision maker experiences an affective reaction, called a valence and denoted $v(t)$, to the consequences (i.e., wins, losses) produced by the selection on trial $t$. Formally, this valence is represented by a weighted average (thus, $w$ ) of the losses and wins produced by the chosen deck:

$$
v(t)=w \cdot \operatorname{loss}(t)+(1-w) \cdot \operatorname{win}(t) .
$$

The loss weight parameter indexes the relative attention or salience of the decision maker to the loss versus the win experienced with each selection. For example, cocaine abusers may persist in choosing from the disadvantageous decks because they are insensitive to the large losses or hypersensitive to the wins produced by these decks. Smaller values of $w$ indicate lower sensitivity to losses.

The update rate parameter, $\boldsymbol{a}$. On the basis of past experience, the decision maker forms expectations about the consequences delivered by each deck. Whenever a deck is chosen, the newly experienced valence produced by that choice modifies the expectancy for that deck. Formally, the new expectancy for deck $j$ after trial $t$, denoted $E v\left(D_{j} \mid t\right)$, is a weighted average of the previous expectancy, $E v\left(D_{j} \mid t-1\right)$, and the newly experienced valence, $v(t)$ :

$$
E v\left(D_{j} \mid t\right)=a \cdot v(t)+(1-a) \cdot E v\left(D_{j} \mid t-1\right) .
$$

The update rate parameter, $a$, determines the relative influence of the new experience on the expectancies. Large values of $a$ indicate strong recency effects and more rapid forgetting of past outcomes. Small values of $a$ indicate the persistence of influences over longer spans of selections. For example, cocaine abusers may have a high learning rate, which would cause them to forget the infrequently occurring large losses produced by these decks, and thus to continue choosing disadvantageously.

The sensitivity parameter, $\theta$. The decision maker's choice on each trial is based not only on the expectancies for each deck, but also on the consistency with which the decision maker applies those expectancies when making the selections. This consistency is moderated by the sensitivity parameter, denoted $\theta$. The probability of choosing a deck is assumed to be a ratio of the strength of that deck relative to the sum of the strengths of all the decks:

$$
P[\text { choose deck } j]=S_{j} /\left(S_{\mathrm{A}}+S_{\mathrm{B}}+S_{\mathrm{C}}+S_{\mathrm{D}}\right) .
$$

Furthermore, the strength of a given deck is determined by the expectancy for that deck multiplied by the sensitivity parameter, $\theta(t)$ as follows:

$$
S_{j}=\exp \left[\theta(t) \cdot E v\left(D_{j} \mid t\right)\right] .
$$

When the sensitivity is very low, choices are inconsistent, random, reckless, impulsive, and independent of the expectancies; when sensitivity is very high, the deck that has the maximum expectancy will almost certainly be chosen. For example, cocaine abusers may fail to select a high proportion of advantageous cards because they respond unreliably to their expectations, and their choices are mostly random guesses, an effect that would reflect lower values of consistency. The sensitivity parameter $\theta(t)$ is assumed to change across trials according to a power function $\theta(t)=\left(t^{c / 10}\right)$.

Parameter values are estimated separately for each participant by solving for the parameter values that, according to the model, maximize the likelihood of each participant's observed choices from all four decks across the 250 trials. The expectancy valence model was compared with each baseline model by computing the improvement index: $G^{2}=2(\log$ likelihood expectancy valence model $)-2(\log$ 
likelihood baseline model). Thus, for each participant, the cognitive modeling process yields a model comparison index and three parameter estimates of the three hypothesized psychological processes that influence choice behavior. These parameter estimates can be used as additional individual difference measures and correlated with other measures such as age, IQ, and severity of drug use.

To evaluate the validity of the expectancy valence model, it was compared with two different baseline statistical models. The random baseline model assumes that the selection from each deck is random with a .25 probability throughout training. The constant baseline model assumes that the probability of choosing from each deck remains constant across trials, but this probability is directly estimated from each participant's 250 choice trials. ${ }^{2}$

\section{RESULTS}

\section{Gambling Task Performance}

The comparison group gradually learned to choose from the advantageous decks, whereas the cocaine group chose nearly equally from the advantageous and disadvantageous decks (see Figure 1, jagged curves). The cocaine group selected significantly fewer advantageous cards [analysis of covariance, $F(1,22)=7.8, M S_{\mathrm{e}}=2.7$, $p=.01]$. This group effect remained after covarying out the effects of age and IQ.

\section{Cognitive Modeling Results}

Figure 1 shows the choice predictions derived from the expectancy valence model (smooth curves) with the observed proportion of choices (jagged curves). The model was fit to all four choices, whereas the figure, for simplicity, shows only the proportion of advantageous choices. As can be seen in the figure, the expectancy valence model tracks the observed trends fairly closely. The percentages of variance $\left(R^{2}\right)$ predicted by the model for the data points shown in the figure were $82 \%$ and $30 \%$ for the comparison and cocaine-abuse groups, respectively.

Comparison with the random baseline model. The average $G^{2}$ improvement of the expectancy valence model over the random baseline was 144.67 and 45.79 for the comparison and cocaine-abuse groups, respectively. The expectancy valence model has three parameters, and the (nested) random baseline model has no parameters. The critical value for a chi-square significance test of the $G^{2}$ improvement $(3 d f, p<.05)$ equals 7.81 . Ninety-two percent of the participants (13 of 14 comparison subjects, and 11 of 12 cocaine abusers) had statistically significant improvements in $G^{2}$, indicating improved fit of the expectancy valence model over the random baseline model.

Comparison with the constant baseline model. The average $G^{2}$ improvement of the expectancy valence model over the constant baseline model was 55.99 and 5.27 for the nonabuse and cocaine-abuse groups, respectively. These models are non-nested, and both models have three parameters, so chi-square tests were inappropriate. Instead, a conservative sign test was performed. Eighty-

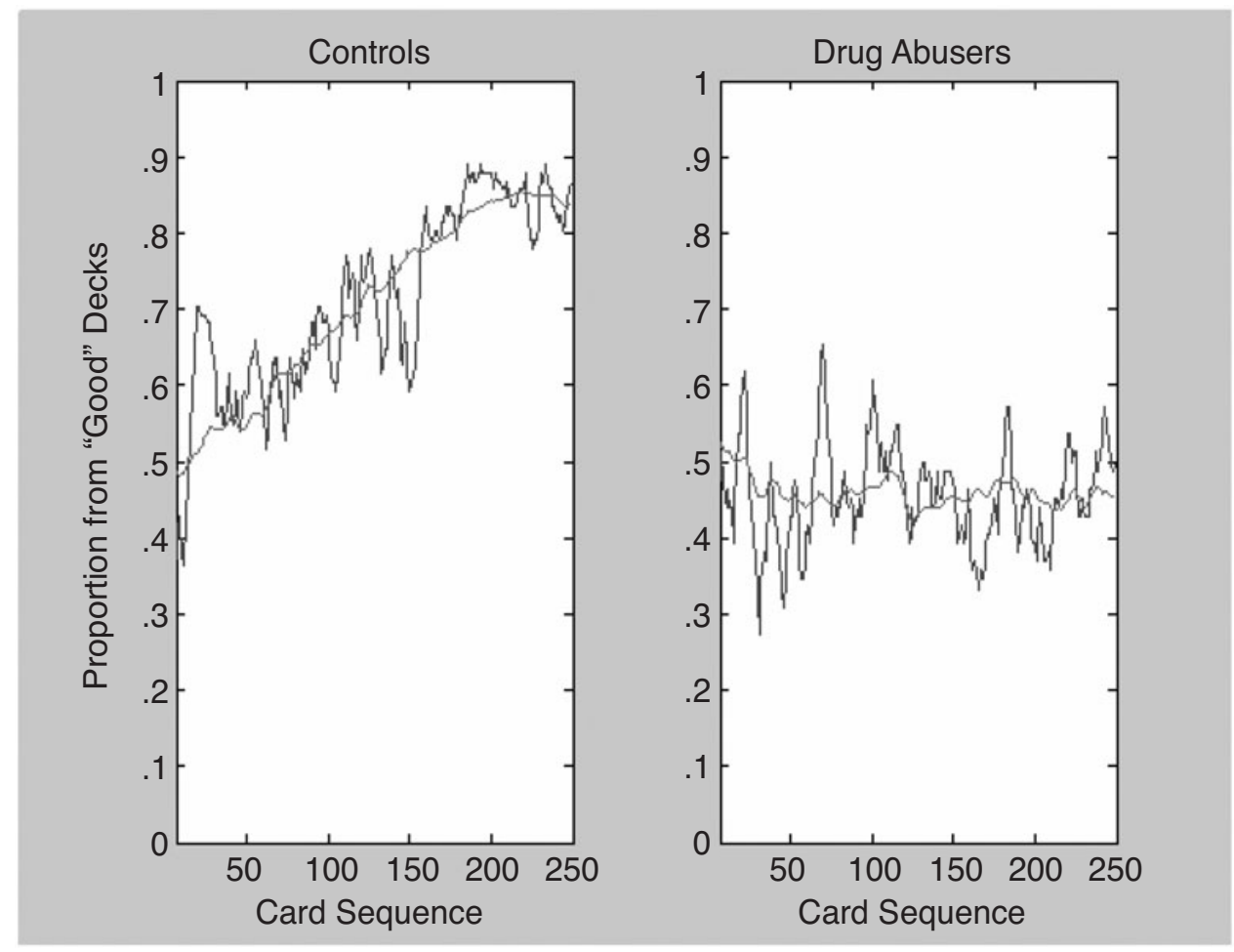

Figure 1. The left and right panels display the results for the comparison and drug-abuse subjects, respectively. Each panel shows the proportion of participants choosing from the advantageous decks as a function of the number of cards selected. The jagged curve represents the actual data, and the smooth curve represents the predictions of the model. Both curves have been linearly filtered by a moving average window of size 7 . 
one percent of the participants (13 of 14 comparison and 8 of 12 cocaine-abusing participants produced positive $G^{2}$ fit statistics, indicating improved fit of the expectancy valence model over the constant baseline model, which was significantly greater than expected by chance $(z=$ $3.14, p<.01)$. For the drug-abuse group, a final check was performed by statistically testing the improvement using a new test for non-nested models (observed $z=$ $1.65, p=.05$, one-tailed; see Golden, 2000). ${ }^{3}$

\section{Group Comparisons on Model Parameters}

A comparison of the groups on the three model parameters indicated group effects on the loss weight and sensitivity, but not updating rate parameters (see Table 1). Although the mean updating rate parameter did not differ significantly across the two groups $[t(17.41)=-.977$, $p=.34]$, the variance within for the cocaine-abuse group was significantly larger than that for the comparison group ( $F=6.77, p=.016$, Levene's test). The loss weight parameter was significantly lower for the cocaineabuse group than for the comparison group $[t(24)=$ $3.40, p=.002]$, indicating that the card selections of the cocaine-abuse group were less influenced by losses (or more influenced by gains) than were those of the comparison group. The sensitivity parameter for the cocaine abusers was significantly smaller than that for the comparison group $[t(24)=2.53, p=.018]$, indicating that the card selections of the cocaine abusers were less consistently related to their expectancies. Further analyses after dropping the 4 poorly fit participants from the drugabuse group (those with negative $G^{2}$ relative to the constant baseline model), yielded the same statistical conclusions. In addition, we computed an analysis of covariance with age and IQ as the covariates. Results showed that these group differences in model parameters were not explained by the group differences in age and IQ; a significant group effect remained on the loss weight parameter, there was a trend in the sensitivity parameter, but there was no apparent difference in the update rate parameter. ${ }^{4}$

\section{Association of Model Parameters \\ With Age and IQ}

Separate multiple regression analyses were performed to determine the magnitude of the independent effects of age and IQ on each of the model parameters. Neither age nor IQ had significant effects on the update rate parameter. For the loss weight parameter, although IQ was not signif- icant, there was a marginal effect of age $(\beta=-.42, S E=$ $.21 ; t=-2.00, p=.057)$. For the sensitivity parameter, although age was not significant, there was a significant effect of IQ $(\beta=.54, S E=.20 ; t=2.72, p=.012)$.

\section{Utility of Model Parameters for Predicting Group Membership}

A discriminant function analysis, used to determine the extent to which the three parameters could predict group membership, was statistically significant (Wilks's $\lambda=.506, \chi^{2}=15.2, p=.002$ ). All of the drug abuse participants were correctly classified, and 11 of the 14 nonabusers were correctly classified; so the overall rate of correct classification was $89 \%$. The loss weight parameter produced the largest standardized coefficient (.88), the sensitivity parameter was next (.71), and the update rate parameter contributed very little $(-.05)$. Even when we included age and IQ into the discriminant function analysis, the weight parameter continued to produce the largest standardized coefficient $(-.42$ for age, .51 for IQ, .078 for the update rate parameter, .73 for the weight parameter, and .53 for the sensitivity parameter).

\section{DISCUSSION}

\section{Basic Findings}

Cocaine abusers failed to learn to choose from the advantageous decks in the Iowa gambling task, whereas comparison subjects rapidly learned to do so. The expectancy valence model provided a better fit than did two baseline models for a majority of the participants. The present study identified which of three possible psychological explanations were responsible for this deficit. The parameter estimates obtained from the expectancy valence model yielded the following conclusions. First, the decision making of the cocaine abusers on the task was less responsive to losses (or more responsive to gains) than was the decision making of the comparison group. This suggests that differences in motivational processes are a likely source of the differences in the decision-making styles of cocaine abusers, which is consistent with several major accounts in the drug abuse literature (Bechara \& Damasio, 2002; Bechara et al., 2002; Grant et al., 2000; Kirby et al., 1999; London et al., 2000). Second, the choices of the cocaine group were more random and less consistently related to expected payoffs than were the choices made by the comparison

Table 1

Summary of Parameter Estimates From the Expectancy Valence Model

\begin{tabular}{|c|c|c|c|c|c|c|c|c|c|}
\hline \multirow[b]{2}{*}{ Group } & \multicolumn{3}{|c|}{ Loss Weight } & \multicolumn{3}{|c|}{ Update Rate } & \multicolumn{3}{|c|}{ Sensitivity } \\
\hline & $M^{*}$ & Med. & $S D$ & $M \dagger$ & Med. & $S D$ & $M \ddagger$ & Med. & $S D$ \\
\hline Control & .63 & .67 & .28 & .23 & .06 & .42 & 1.58 & 1.30 & 1.95 \\
\hline Drug & .25 & .25 & .29 & .45 & .04 & .70 & -.09 & .18 & 1.29 \\
\hline
\end{tabular}

$*_{t}(24)=4.3, p=.002 . \quad \dagger t(24)=-1.02, p>.15 . \quad \ddagger t(24)=2.53, p=.018 . \quad$ The tests of loss weight were based on estimates of $w$ in Equation 1, the tests of update rate were based on the parameter $a$ in Equation 2, and the tests of sensitivity parameter were based on the coefficient $c$ in the relation $\theta(t)=(t / 10)^{c}$ used in Equation 3B. 
group. This indicates that the response process also contributes to the decision-making differences between cocaine abusers and the comparison group. In contrast to the motivational processes and choice consistency, the learning/memory processes did not distinguish the drug abusers from the comparison group.

\section{Comparison With Previous Cognitive Modeling Results}

Busemeyer and Stout (2002) conducted cognitive modeling analyses of Huntington's participants on the Iowa gambling task. Huntington's disease is a progressive degenerative neurological disease associated with declines in cognitive function - particularly memory, executive, and attentional functions. The decision-making deficit exhibited by the Huntington's disease group was explained by the update rate parameter and was not associated with the loss weight parameter, which did not differ between the Huntington's and comparison groups. The results from the Huntington's disease study are in sharp contrast with this study of cocaine abusers because the latter points strongly to motivational differences as the source of the deficit in cocaine abusers. The findings in these studies have some external validity. For example, additional evidence points to learning/memory as a likely explanation for poor performance in Huntington's disease. These individuals have a dementing disease, and memory impairment is by definition an invariant of dementia. Also, in Stout, Rodawalt, and Siemers (2001), performance on the gambling task was associated with other measures of learning and memory. In contrast, the finding that motivation, or the attention given to losses relative to gains, is a stronger determinant in the decision process of the cocaine abusers is consistent with the conclusions from other studies of drug abusers (Bechara \& Damasio, 2002; Bechara et al., 2002; Kirby et al., 1999; Petry et al., 1998). ${ }^{5}$ Interestingly, the sensitivity parameter was lower for the clinical groups in both the Huntington's study and the present study of cocaine abusers. This is consistent with the possibility that reduced consistency in choice behavior may be a less specific feature in neurobehavioral disorders.

The comparison of the Busemeyer and Stout (2002) study and the present study illustrates an important point about what can be gained from applying a formal model to decision data from clinical groups. That is, although the behavioral results from the cocaine abusers in the present study and the Huntington's participants in the previous study appear similar, these performance deficits have different underlying theoretical explanations (i.e., motivational vs. learning, respectively). Such differences can be revealed and systematically studied with a cognitive modeling approach. This may be particularly valuable in describing subgroups of clinical populations that show similar deficits with different underlying causes. The parameter estimates can also be studied in conjunction with other individual difference variables, such as personality features or the extent of drug use.

\section{Utility of Cognitive Modeling for Studies of Drug Abuse}

The use of cognitive modeling to study psychological processes provides a means for building a bridge between neurophysiology and behavior. Neuroscience research has found that drug abuse damages regions of the brain, such as the nucleus accumbens, which are responsible for processing reinforcement. However, this damage could result in either learning or motivational deficits or both. Without our cognitive modeling analysis, it would not be possible to discern whether the performance deficits in cocaine abusers in this study were due to learning/memory or motivational processes. If our results had indicated that learning/memory was responsible for the performance deficit, that finding would lead to the investigation of neurophysiological mechanisms of learning and memory. Instead, our cognitive modeling analysis identifies a motivational source for the behavioral deficit. This finding builds theoretical support for research targeting the brain's motivational systems in studies of drug abuse.

\section{REFERENCES}

Bechara, A., \& Damasio, H. (2002). Decision-making and addiction (Part I): Impaired activation of somatic states in substance dependent individuals when pondering decisions with negative future consequences. Neuropsychologia, 40, 1675-1689.

Bechara, A., Damasio, A. R., DAMASio, H., \& Anderson, S. W. (1994). Insensitivity to future consequences following damage to the human prefrontal cortex. Cognition, 50, 7-15.

Bechara, A., Dolan, S., \& HiNDES, A. (2002). Decision-making and addiction (Part II): Myopia for the future or hypersensitivity to reward? Neuropsychologia, 40, 1690-1705.

BusemeYer, J. R., \& STOUT, J. C. (2002). A contribution of cognitive decision models to clinical assessment: Decomposing performance on the Bechara gambling task. Psychological Assessment, 14, 253-262.

Golden, R. M. (2000). Statistical tests for comparing possibly misspecified and non-nested models. Journal of Mathematical Psychology, 44, 153-170.

GRANT, S., Contoreggi, C., \& London, E. D. (2000). Drug abusers show impaired performance in a laboratory test of decision making. Neuropsychologia, 38, 1180-1187.

Kirby, K. N., Petry, N. M., \& BicKel, W. K. (1999). Heroin addicts have higher discount rates for delayed rewards than non-drug-using controls. Journal of Experimental Psychology: General, 128, 78-87.

London, E. D., ERnst, M., Grant, S., Bonson, K., \& Weinstein, A. (2000). Orbitofrontal cortex and human drug abuse: Functional imaging. Cerebral Cortex, 10, 334-342.

Maddox, W. T., \& Filoteo, J. V. (2001). Striatal contributions to category learning: Quantitative modeling of simple linear and complex nonlinear rule learning in patients with Parkinson's disease. Journal of the International Neuropsychological Society, 7, 710-727.

MAZAS, C. A., Finn, P. R., \& SteInMETZ, J. E. (2000). Decision-making biases, antisocial personality, and early-onset alcoholism. Alcoholism: Clinical \& Experimental Research, 24, 1036-1040.

Petry, N. M., Bickel, W. K., \& ArNetT, M. (1998). Shortened time horizons and insensitivity to future consequences in heroin addicts. Addiction, 93, 729-738.

RATCLIFF, R., THAPAR, A., \& McKoon, G. (2001). The effects of aging on reaction time in a signal detection task. Psychology \& Aging, $\underline{\mathbf{1 6}}_{2}$ 323-341

RiEfER, D. M., KNAPP, B. R., Batchelder, W. H., Bamber, D., \& Manifold, V. (2002). Cognitive psychometrics: Assessing storage and retrieval deficits in special populations with multinomial processing tree models. Psychological Assessment, 14, 184-201.

Rogers, R. D., Everitt, B. J., Baldacchino, A., Blackshaw, A. J., 
Swainson, R., Wynne, K., Baker, N. B., Hunter, J., Carthy, T., Booker, E., London, M., Deakin, J. F. W., Sahakian, B. J., \& RobBINS, T. W. (1999). Dissociable deficits in the decision-making cognition of chronic amphetamine abusers, opiate abusers, patients with focal damage to prefrontal cortex, and tryptophan-depleted normal volunteers: Evidence for monoaminergic mechanisms. Neuropsychopharmacology, 20, 322-339.

Stout, J. C., Busemeyer, J. R., Bechara, A., \& Lin, A. (2002). Cognitive modeling of decision making in a simulated gambling task in frontal or somatosensory cortex damage [Abstract]. Journal of Cognitive Neuroscience, 41 (Suppl.), 75.

Stout, J. C., Rock, S. L., CAmpbell, M. C., Busemeyer, J. R., \& Finn, P. R. (in press). Psychological processes underlying risky decisions in drug abusers. Psychology of Addictive Behaviors.

Stout, J. C., Rodawalt, W. C., \& Siemers, E. R. (2001). Risky decision making in Huntington's disease. Journal of the International Neuropsychological Society, 7, 92-101.

ZACHARY, R. (1986). Shipley Institute of Living Scale. Los Angeles: Western Psychological Services.

ZAKI, S. R., \& NOSOFSKY, R. M. (2001). Exemplar accounts of blending and distinctiveness effects in perceptual old-new recognition. Journal of Experimental Psychology: Learning, Memory, \& Cognition, 27, 1022-1041.

\section{NOTES}

1. We recently replicated the results reported in the present study, using real rather than play payoffs (Stout, Rock, Campbell, Busemeyer, $\&$ Finn, in press) comparing drug abusers and nonabusers. The real payoffs stated in this article were divided by 100 .

2. We also compared the expectancy valence model with two other cognitive models presented in Busemeyer and Stout (2002). The mean improvements in $G^{2}$ of the expectancy valence model over the strategy switching model were 39.77 and 16.30 for the comparison and drugabuse groups. Mean improvements in $G^{2}$ of the expectancy valence model over the Bayesian expected utility model were 39.77 and 16.30 for the comparison and drug-abuse groups.

3. Rock et al. (2003) found approximately the same $G^{2}$ improvements for the expectancy valence model, replicating the present findings.

4. Rock et al. (2003) replicated the differences in parameter estimates reported in the article.

5. More recently, Stout, Bechara, Busemeyer, and Lin (2004) conducted cognitive modeling analyses of data from orbital frontal cortex damaged patients, and the results match those obtained from the cocaine abusers.

(Manuscript received April 30, 2003; revision accepted for publication July 29, 2003.) 